\title{
CREDIBILITY MEASURE IN TWEET RETRIEVAL BASED ON TEXTUAL CONSISTENCY
}

\author{
Ibtissem Mejbri and Lobna Hlaoua \\ Mars Research Lab, Isitcom, University of Sousse, Hammam Sousse, Tunisia
}

\begin{abstract}
Nowadays, social media is a space where information can be shared concerning any type of event. These networks have become a mass of social information that is accumulated day by day. This accumulation can even be doubled in a time of sudden crisis or even within the spread of a pandemic as the example of the famous COVID-19 virus. This social information can, without a doubt, impact, not only the internet surfer, but also the others who have no relationship with the social networks. The major problem here is based on the credibility of information, since the users have the ability of sharing any kind of information. This, in its way, explains why the credibility of social information presents an important challenge. Consequently, in this paper, we are particularly interested in the social media Twitter, where our primary goal is to evaluate the credibility score of a tweet. More precisely, we have presented a hybrid system that takes into consideration, on one hand, the content and the author of the tweet, and on the other hand, includes the notion of textual coherence between the tweet and its comments. In order to validate our proposed approach, we performed our work on a database based on the tweets concerning the COVID-19. Experimental study shows the efficiency of our system, since it's able to reach the same level of judgment as a human. The advantage of our approach based on adapting to changes, where each comment added can also influence credibility, unlike most of the work which is based on machine learning.
\end{abstract}

\section{KEYWORDS}

Credibility, Tweet Retrieval, Comments, Textual Consistency

\section{INTRODUCTION}

The search of information has grown since the appearance of Web 2.0, more precisely since the emerge of the social media (Facebook, Twitter, LinkedIn...). This new innovation is viewed as the most useful and rich new source of information. As the days go by, the number of users increases in an exceptional manner. According to the latest statistics ${ }^{1}$, we found 3.484 million users in 2019 . Social media evaluates the information retrieval permitting each surfer to share, in an instant manner, millions of information tackling different subjects. The famous example, Twitter, the microblogging media, today presents an excellent information radiator tool in real time, since it aims to shed light on the potential of this social research. In fact, the main goal of this media consists of perfectly responding to the needs of the users based on the diversity of information that it possesses with approximately 500 million tweets sent per day. Evidently, in phase of this growth of information shared each day, the information retrieval problem does no longer exist. Nevertheless, the main problem revolves around the quality of information. In other words, the majority of times rumors possess strong impact that can in certain cases affect the public opinion and especially when it tackles sensitive subjects (Scientific, meteorological, political etc.). As an example, the millions of tweets shared concerning the disease provoked by the coronavirus, the virus that terrified humanity, without knowing whether they are reliable or not. In that matter, today we talk about a massive quantity of brilliant data and also filled with rumors. According to the study of (Zhao et al., 2015), most microblogging sites are filled with one third of false information. In this order the social media have become, unfortunately, in a certain manner, a field for rumors (Azri et al.,2019). For this reason, the credibility presents a subject of interest and actuality. So, it is evident before sharing any information, the system has to be able to measure its credibility. As a matter of fact, many works are situated in the detection of rumors field in Twitter and a

\footnotetext{
${ }^{1}$ https://www.smartinsights.com/social-media-marketing/social-media-strategy/new-global-social-media-research/
} 
very good part of the approach is interested in the score calculation of credibility, but it still always non-sufficient since at any given moment anyone, behind their little screen, can say whatever they feel like saying according to any event (Froissart et al., 2002). In order to fill the gaps and define the credibility score to obtain an optimal score and precise each tweet a user issues, in this paper, we have the opportunity to propose a hybrid approach. This approach is based partly on the aggregation of three levels of credibility, well explain thereafter, and partly on the analysis of comments with the notion of " Textual consistency", since our definition of credibility in this paper, is strongly related with the reasoning of the human being based, on the comments associated with each tweet.

This paper is organized as follows: Starting with section 2, we presented related credibility estimation works. Moving on to section 3 and 4 with the different levels of our approach as well as the different evaluations results. Finally, we conclude this paper with a conclusion and our future work.

\section{RELATED WORKS}

In order to enhance the performances and to obtain a credible optimal score, according to literature, there is a number of works that evaluate the credibility of the tweet. (Alrubaian et al., 2018) (Nagy et al., 2012) classify the credibility into big levels: "Post Level", "User Level", "Topic Level" and "Hybrid-Level" which combine the three levels at the same time, where each level has their own priorities, characteristics, advantages and disadvantages.

\section{- Post-Level}

The principle of this level is founded essentially on the analysis of the attributes of the tweet's content. In other words, this level is not interested neither in the writer nor the subjects tackled by the tweet. But on the opposite, it sheds light only on the content of the post, which means the length of the tweet, the number of words, if it contains pictures and videos (Boididou et al., 2015). So, the more the tweet contains these attributes, the more it has a more important and elevated value of credibility. Based on these functionalities, (Sharf et al., 2013) have proposed an android application dedicated to measure the credibility of current information on Twitter that provide the user with a graphic representation that expresses the credibility rate.

- User-Level

Other researchers supposed that the credibility of the tweet is strongly related to the credibility of the contained source. In that order, many works are interested in knowing the influential users and relying on the reputation of the social media. To attend this goal, it is important to extract the characteristics from the account (Alrubaian et al., 2018) in order to identify the importance of the user following their number of followers and followed accounts, their picture, if the account is verified or not... (Westerman et al., 2012) have elaborated a study only available information elements that can influence and impact the credibility of the source.

\section{- Topic-Level}

The works of (Damak et al., 2013) have announced the record of 500 million tweets published per day. This quantity can be doubled when a trending subject or an important topic spread (Al-Qurishi et al., 2017). In fact, when users begin to comment and share their opinions, at times being totally different, it can either confirm or deny the topic. For this reason, an acceptable number of approaches have taken the occasion to estimate the efficiency and flexibility of information carrying topics with strong impact.

\section{- Hybrid-Level}

Certain researchers have adapted hybrid approach by using the two or even three levels together and their features. The aggregation of the three levels then to assemble the functionalities and the strong sides of each level (Canini et al., 2010). In a general manner, the hybrid approaches provide powerful results and performances compared to the others. As a result, many researchers work on this level since it solves the obstacles that prevent the analysis of the credibility. (Lorek et al., 2015) proposed a combined approach using both content and feature analysis. This approach achieves an accuracy of 89\% (the accuracy increases between $8 \%$ and $10 \%)$.

Each level possesses advantages and disadvantages. Indeed, it is difficult to judge credibility only from a tweet of 280 characters or from the user reputation, since from Twitter follower markets users can obtain followers.

In a general manner, the hybrid level is able to combine all the advantages and can produce better results.

However, in this level the comments were not taken into consideration although they may be a new source of evidence. 


\section{CREDIBILITY ASSESSMENT BASED ON THE CONSISTENCY OF REPLAY}

\subsection{Motivation}

Firstly, the idea of using comments is presented among the most dominant factors in this strategy to test the importance of the blog. Secondly, the comments in the e-commerce domain it obtains a preponderant effect on the reputation of the products. According to these facts, in this paper, we have chosen to highlight this notion. We are particularly interested in the combined levels of credibility and shedding light on the different opinions of the users throughout the comments that are most of the time ignored in related works. This choice is not random, but very well studied because of its advantages precisely in the degree of impact of the comments on news, decisions, products... Also, the comments can without a doubt clarify the ideas and opinions. But, it still to be known if a tweet is credible or not by observing the coherence of its comments?

\subsection{Description of the Approach}

To perfectly respond to the previous question, we have proposed an approach that is mainly based on the consistency of the comments, in order to well define its impact on the credibility measure. To make the access fast and effective, our first step is based on the representation of the tweet. Said differently, this task consists in extracting the most important information in creating two vectors; The first one being a logical view of the tweet, while the second one indicates another logical view dedicated for the author that designates its position in the social network. The tweet's table is defined throughout different fields such as "tweet author", "content", "number of shares" " number of responses "... When it comes to the author's table, it consists of fundamental fields for example "user name ", "number of followers", "number of the following accounts"... The main goal of our approach, is to obtain a vague score of credibility not only throughout the level of the tweet content and the author level but also throughout the comments. That's why, it's important to extract and represent the comments. So, this step possesses the same objective, but it needs the addition of another specific field permitting to identify the associated tweet "parent tweet" with a given tweet. Our approach is based on 4 steps as presented in figure 1 and explained below.

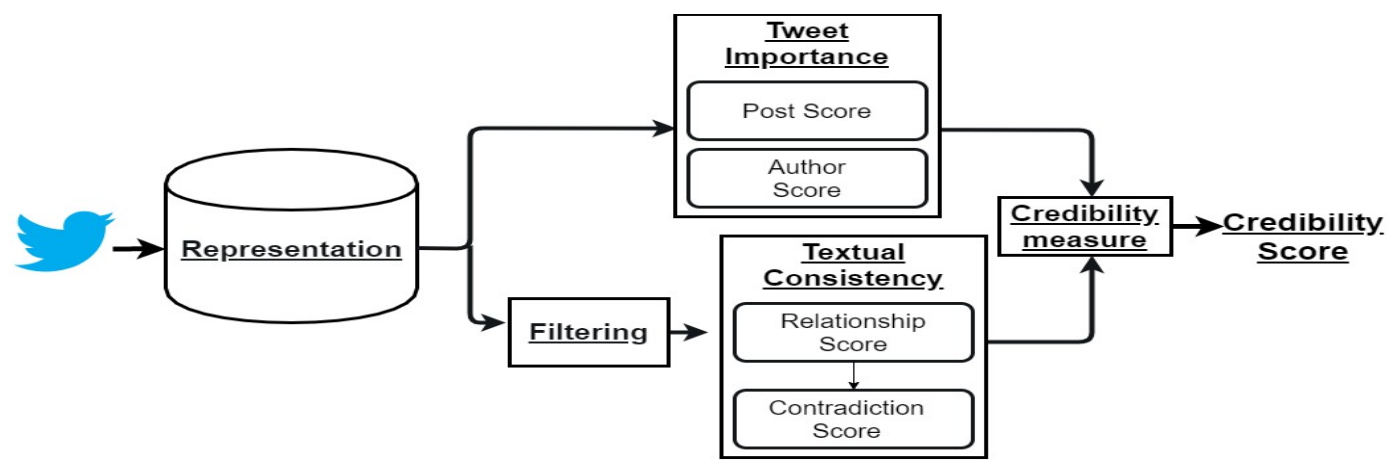

Figure 1. Process for calculating the credibility of a tweet

\subsection{Filtering}

As a side note, it is important to mention that in our case, we are only interested in the English language. To obtain a tweet with relevant and precise comments it is essential to go through the filter comments step in order to reduce the noise and only show the high-quality comments. It means, in this step, we are partly interested in eliminating all the comments that have different languages than English. And also, we are only keeping the comments that carry a clear idea. So, we will erase all the comments with grammatical, syntax or autographic mistakes that can without a doubt trouble the relevant credibility. 


\subsection{Tweet Importance}

In a general manner, a tweet is defined as important, in the first place, if its content is rich and possess a number of acceptable interactions. And as second place, if it is written by an author with a good social reputation. In order to calculate this score, our proposition goes through two levels. The first being interested in the importance of score in the level of the post, the second being interested in the score in the level of the author as it shows in the formula below:

$$
\text { TweetImportanceScore }(t, u)=\text { AuthorScore }(u) * \operatorname{PostScore}(t)
$$

As a start, we begin with the first level, where the calculation is based on the analysis of the content of the attributes as well as the volume of the shares, the responses, the likes... The more these characteristics are presented, the more the importance of the score is increased.

$$
\begin{aligned}
& \operatorname{PostScore}(t)=\frac{(\operatorname{length}(t)+\operatorname{retweet}(t)+\operatorname{replay}(t)+\operatorname{like}(t)+\operatorname{Url}(t)+\operatorname{Hashtag}(t)+\operatorname{Mention}(}{7} \\
& \operatorname{Url}(t)=\left\{\begin{array}{rr}
1 & \text { if t contains } U R L \\
0 & \text { Otherwise }
\end{array}\right. \\
& \operatorname{Hashtag}(t)=\left\{\begin{array}{l}
1 \\
0
\end{array} \quad\right. \text { if t contains Hashtag } \\
& \operatorname{Mention}(t)=\left\{\begin{array}{lr}
1 & \text { if t contains Mention } \\
0 & \text { Otherwise }
\end{array}\right.
\end{aligned}
$$

The calculation of the second level is founded upon the extracted characteristics from the author's account (u), which reflects its reputation in the social network. Our proposed formula is calculated as follows:

$$
\begin{gathered}
\operatorname{AuthorScore}(u)=\frac{(\text { followers }(u)+\text { following }(u)+\text { likesCount }(u)++ \text { verifiedAccount }(u))}{4} \\
\text { verifiedAccount }(u)= \begin{cases}1 & \text { if Account is verified by Twitter } \\
0 & \text { Otherwise }\end{cases}
\end{gathered}
$$

\subsection{Textual Consistency}

This step is the heart of our process, the textual coherence or consistency notion (Bergeron et al., 2003) where we will know if there is a chain of information between the tweet and its comments. (Charolles 1978) consider that the textual coherence is assured throughout 4 main rules (Progression, Non-contradiction, Repetition and Relation). It is true that these four rules granite the redaction in a well coherent text, but in our case, we are interested in the coherence of the comments. Meaning, it is useless to apply these four rules in our process. In other words, we will only be interested in the "Relation" and "Non-contradiction". That we can define as follows:

- Relation: This rule focuses on the congruence of information (the events, the actions, and the facts) (Carbonneau et al., 2005). Put differently, to produce a sequence or a coherent text, the sentences brought must establish a meaningful relationship between them and must belong to the same universe (domain).

- Non-contradiction: This rule is among the most essential rules for the construction of the textual coherence. In a general manner the existence of contradictory information in a text does not only disturb the coherence but also the comprehension, the readability and the credibility. In order to avoid these problems, the principle of this rule requires that the succession of information does not suffer from any contradiction with those which proceed.

In that order, the coherence score of each comment is calculated thanks to this following equation:

$\operatorname{TextConsistencyScore}(t, r)=\left\{\begin{array}{c}\text { ContradictionScore }(t, r)+\text { RelationshipScore }(t, r) \quad \text { if ContradictionScore }=1 \\ \text { ContradictionScore }(t, r)-\text { RelationshipScore }(t, r) \text { if ContradictionScore }=-1\end{array}\right.$ 


\section{Relationship score}

Before applying our calculation algorithm of the relationship score, we first begin with eliminating of all the empty words (the, this, so...) in the tweet and its comments. Since these empty words have no influence but can disturb the calculations. Our proposed algorithm to calculate the relationship score is based on the calculation of semantic similarities between the tweet $(\mathrm{t})$ and the comment $(\mathrm{r})$. Differently put, with the help of an online thesaurus we will calculate the number of words in relation. The more this number increases the more the comment is in relation to the tweet. (See algorithm table 1).

Table 1. Relationship Score Calculation Algorithm

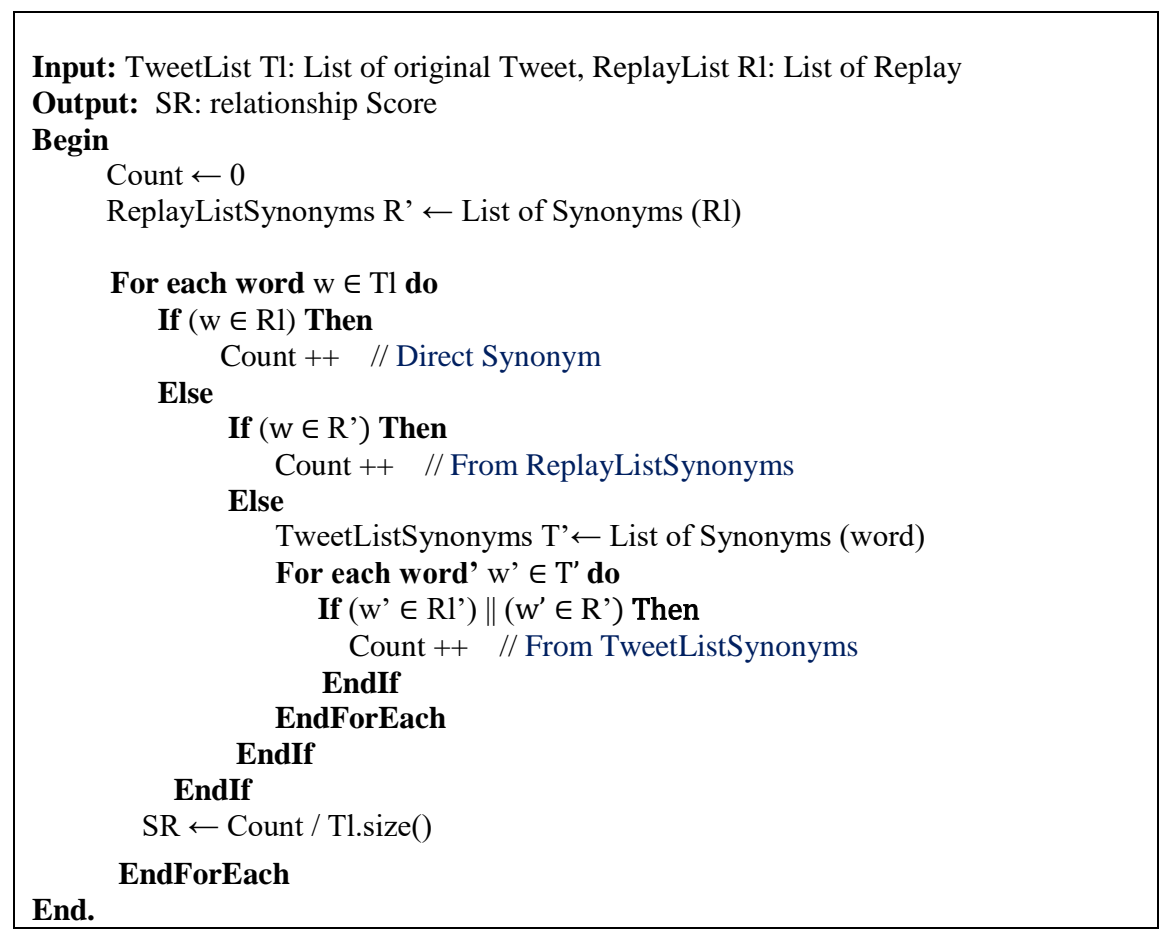

\section{Contradiction score}

The notion of contradiction is the second aspect of our approach. In a general manner, the comments can be divided into two groups. The first group is dedicated to the comments that support and confirm the tweet's idea. The second group concern the opposing comments that contradicts in any manner the tweet. To simplify the idea, we take this example of a tweet $\mathrm{T}$ with two comments $\mathrm{C} 1$ and $\mathrm{C} 2$ below:

- T: Covid-19 is a deadly virus.

- C1: It's wrong, covid-19 is a simple virus, it's not deadly.

- C2: Exactly, one of the most dangerous viruses killing humanity.

In this regard, in order to strictly understand the ownership of each comment, we need the collected indicators which are detailed in section 4.1, where through them we can discover the positioning of the comments. In that order, the contradiction score is calculated as follows:

$$
\text { Contradictionscore }(t, r)=\left\{\begin{aligned}
-1 & \text { if } r \text { contains expressions contradict } t \\
1 & \text { if } r \text { contains expressions confirm } t
\end{aligned}\right.
$$

In the intention of ameliorating our approach and obtain a hybrid system, the integration of a commentary author score can be considered as a predominant factor that can without a doubt influence the calculation. In this context, our idea consists of multiplying the coherence score with the commentary author score as it is indicated in the following formula:

$$
\operatorname{ReplayScore}(t, r, u)=\text { TextConsistencyScore }(t, r) * \text { AuthorScore }(u)
$$


The ReplayScore needs to be recalculated for each comment. In other words, each score obtained is classified whether in the list of comments that support the idea of the tweet named ReplayForScore, or in the list of comments that contradicts the tweet named ReplayedAgainstScore. To further explain, the list of scores that belongs to ReplayForScore are scores strictly positive, on the other hand the list of ReplayAgainstScore is dedicated for the negative scores. At this point, it is important to have a final consistency score which is a combination of the ReplayFor and the ReplayAgainst. The main foal of this score is to assess, in a global manner, to what extent different users are convinced by the credibility of the tweet.

$$
\operatorname{ReplayConsistencyScore}(t, r)=\frac{\sum \operatorname{ReplayForScore}(t, r)}{\mid \sum \text { ReplayForScore }(t, r)|+| \sum \operatorname{ReplayAgaistScore}(t, r) \mid}
$$

\subsection{Credibility Measure}

The goal of this final step is founded on the aggregation of notions in order to obtain a hybrid approach that causes to refine the credibility score that is mainly based on the comments ( $r$ ), the content of the Tweet (t) and the author $(\mathrm{u})$. To obtain this objective, the final calculation of the credibility score is the result of the sum of Tweet's importance score with the final Replay consistency score obtained with the following formula:

$$
\text { CredibilityScore }(t, r, u)=\text { TweetImportanceScore }(t, u)+\operatorname{ReplayConsistencyScore}(t, r)
$$

\section{EXPERIMENTAL EVALUATION}

\subsection{Data Collection}

Tweet collection: In order to validate our proposed solution, in this section, we have focused on solving the major problem of many approaches, we are concerned with the task of collecting real data. Since there is no standard database for the detection of the tweets credibility. So, the majority of works used synthetic data (Stattner, 2012) as a cause of the complexity and difficulty of this task. The importance of our work lies in the choice of tweets. In other words, the selected tweets must belong to the subject of strong impact precisely being on the COVID-19 virus. For this reason, in our case the task of selecting tweets must be manual. Therefore, we used data representing actual tweets, were our test sample established from the official API offered by Twitter and thanks to the Java library Twitter4J. We have collected 200 tweets. Each tweet is associated with its author, it's comments, and the authors of the comments. In this case, in our database we have 4850 comments and 4900 authors. As a consequence, we notice that an author, of our collection, and not only comment numerous times but also write multiple tweets. In order to test if our system is able to detect the credibility as a user (section 4.2), it is crucial for us to create a well-organized form containing all the tweets and necessary information for the tweets and send it to a group of users ( 200 researchers ) to vote which, according to them, the tweets that are credible and which are non-credible.

Adverbs of affirmation and doubts: Taking into consideration the different views of numerous users from the comments is the added aspect to our approach. In which, we are in need of a collection of expressions of approval and disapproval. In fact, we have allocated a first file that contains nearly the majority of the most used expressions that express the approval in Twitter. For example, "It's very true"," That's so true"... And a second file is reserved for the collected expressions of disapproval. For example, "It's fake", "It's a lie"...

\subsection{Experiments and Results}

The main goal of this section is to show the performance of our system, and also to well determine if the idea of aggregating the different levels of credibility, already explained in the section 2 , with the notion of textual consistency can refine the credibility of the tweet. The evolution of our system is performed by the three 
evaluative metrics (Recall, Precision, F-Measure). To put it differently, in order for our approach to be performant, it is necessary for it to be able to ameliorate not only the Precision but also the Recall. Our evaluation strategy is composed of different versions of credibility measure well explained in the following. To well validate our system, it is highly important to compare the scores obtained with the reality (the exact results). Taking it from this point of view, we have chosen to follow the topics of COVID-19 in order to verify its truth, which will allow us to obtain truthful results to exercise the comparison to.

\subsubsection{Impact of Textual Consistency}

In the first version of credibility measure "Tweet importance", we have precisely focused on the Tweet importance score expressed in formula 3.1. In the second version "Textual consistency credibility", we are particularly interested in the textual coherence score between the tweet and its comments expressed in formula 3.8. The main objective of this version, is to study the impact of this notion that adapts the "Relation" factor and the "Non-contradiction" factor to distinguish between the credible and the non-credible tweets. While, in "User consistency credibility", we did not only shed light on the textual coherence but also on the author of each comment expressed in formula 3.10. The concept of this version is based on a simple idea: having a comment coherent with the tweet but written by an author with a mediocre reputation can certainly affect the credibility degree. As the goal is to push the limits, the fundamental aim of the "Credibility assessment system" is to test the impact of our hybrid approach expressed in formula 3.12. Our approach combines, on one hand, the tweet important score, that adapts the publication level with the user level, and on the other hand, the textual coherence score, which takes into consideration the author of the comment.

Table 2. Obtained Results for the credibility measure

\begin{tabular}{lcccccc}
\hline & \multicolumn{3}{c}{ Credible } & \multicolumn{3}{c}{ Non-Credible } \\
\hline & Recall & Precision & F-Measure & Recall & Precision & F-Measure \\
\hline Tweet importance & $61 \%$ & $67 \%$ & $58 \%$ & $28 \%$ & $33 \%$ & $30 \%$ \\
Textual consistency credibility & $84 \%$ & $73 \%$ & $78 \%$ & $42 \%$ & $50 \%$ & $49 \%$ \\
User consistency credibility & $76 \%$ & $76 \%$ & $76 \%$ & $57 \%$ & $57 \%$ & $57 \%$ \\
Credibility assessment system & $\mathbf{7 6 \%}$ & $\mathbf{8 3 \%}$ & $\mathbf{7 9 \%}$ & $\mathbf{7 1 \%}$ & $\mathbf{6 2 \%}$ & $\mathbf{6 6 \%}$ \\
\hline
\end{tabular}

The evaluation results demonstrated in table 2 show the impact of each version of credibility measure. The first result show very well the imperfection of "Tweet importance" to detect "credibility" and "noncredibility. Using only the textual consistency to measure credibility allows to obtain remarkable results, but it's unable to distinguishing the non-credible tweets $(\mathrm{F}-\mathrm{Measure}=49 \%)$. On the other hand, when it comes to "User consistency credibility", we noticed that it has a decent, but still mediocre, improvement when detecting the non-credible tweets. This poor improvement can confirm that the reputation of the comment's author is able to influence the calculations. Concerning the results obtained by "Credibility assessment system", shows that our proposed hybrid system ensures a significant improvement, since it positively affects not only the Recall but also the Precision to detect "credibility" and "non-credibility". This improvement shows the efficiency and performance of our hybrid system.

\subsubsection{Comparison between our Results and the User Judgments}

After evaluating our system in the previous versions with certain and verified information, at this stage, it is important to find out if our proposed approach is able to evaluate and detect the credibility such as the user's judgment. Afterwards, we compared their results with the results obtained with our system. Table 3 shows the obtained evaluation outcome. These results have clearly shown that our hybrid system functions nearly as the judgment of users, that being referring to the ion of the Tweet's credibility (F-Measure $=86 \%$ ) and even the detection of the non-credible tweets ( $\mathrm{F}$ - Measure $=80 \%$ ).

Table 3. Evaluation according user judgments

\begin{tabular}{ccccccc}
\hline & \multicolumn{3}{c}{ Credible } & \multicolumn{3}{c}{ Non-Credible } \\
\hline & Recall & Precision & F-Measure & Recall & Precision & F-Measure \\
\hline Credibility assessment system & $\mathbf{9 0 \%}$ & $\mathbf{8 3 \%}$ & $\mathbf{8 6 \%}$ & $\mathbf{7 7 \%}$ & $\mathbf{8 5 \%}$ & $\mathbf{8 0 \%}$ \\
\hline
\end{tabular}


Finally, we can see that our system is able to detect credibility compared to the user judgments better than the reality. Consequently, in general, it can be confirmed that a hybrid system based on the notion of the textual consistency between the tweet and its comments can detect the credibility of tweet.

\section{CONCLUSION}

The concept of the credibility of social information has become essential. In this paper, we have proposed a hybrid information credibility system in the social network Twitter. The added aspect in our system is that it adapts to change, or in some cases, a new comment added can easily change the result. Our approach depends, on one hand, on the hybridization of different levels and on the other hand, on the addition of the textual coherence notion between the comments and the tweet. The experimental evaluation shows that our system is able to distinguishing the credible tweets and also the non-credible tweets, that while being compared to the judgment of the users and the reality. At this point, it is safe to say that these results successfully show the efficiency of our proposed approach. Consequently, we can confirm that comments may be a new source of evidence able to detect credibility. But in order to ameliorate the results, in our future work we will be adapting the other two factors of the textual coherence notion "Progression" and "Repetition", and also, we will be testing our approach on other datasets to compare it with other approaches.

\section{REFERENCES}

Al-Qurishi, M., Hossain, M.S., Alrubaian, M., Rahman, S.M.M. and Alamri, A., 2017. Leveraging analysis of user behavior to identify malicious activities in large-scale social networks. IEEE Transactions on Industrial Informatics, 14(2), pp.799-813.

Alrubaian, M., Al-Qurishi, M., Alamri, A., Al-Rakhami, M., Hassan, M.M. and Fortino, G., 2018. Credibility in online social networks: A survey. IEEE Access, 7, pp.2828-2855.

Azri, A., Favre, C., Harbi, N. and Darmont, J., 2019, October. Vers une analyse des rumeurs dans les réseaux sociaux basée sur la véracité des images : état de l'art.

Bergeron, R., and Blain, R., 2003. La cohérence textuelle. Québec français, vol. 128, pp. 47-47.

Boididou, C., Andreadou, K., Papadopoulos, S., Dang-Nguyen, D.T., Boato, G., Riegler, M. and Kompatsiaris, Y., 2015. Verifying Multimedia Use at MediaEval 2015. MediaEval, 3(3), p.7.

Canini, K.R., Suh, B. and Pirolli, P., 2010. Finding relevant sources in twitter based on content and social structure. In NIPS Workshop.

Carbonneau, C. and Préfontaine, C., 2005. Enseigner et évaluer la cohérence textuelle. Québec français, (138), pp.78-81.

Charolles, M., 1978. Introduction aux problemes de la coherence des textes:(Approche théorique et étude des pratiques pédagogiques). Langue française, (38), pp.7-41.

Damak, F., Pinel-Sauvagnat, K., Boughanem, M. and Cabanac, G., 2013, March. Effectiveness of state-of-the-art features for microblog search. In Proceedings of the 28th Annual ACM Symposium on Applied Computing (pp. 914-919).

Froissart, P., 2002. Rumeurs sur Internet. Les cahiers de médiologie, 13 (premier semestre). Gallimard, Paris, pp. 205-208.

Lorek, K., Suehiro-Wiciński, J., Jankowski-Lorek, M. and Gupta, A., 2015. Automated credibility assessment on Twitter. Computer Science, 16(2)), pp.157-168.

Nagy, A. and Stamberger, J., 2012. Credo: A framework for semi-supervised credibility assessment for social networks. In Proceedings of the International Conference on Data Mining (DMIN) (p. 1). The Steering Committee of The World Congress in Computer Science, Computer Engineering and Applied Computing (WorldComp).

Sharf, Z. and Saeed, A.U., 2013. Twitter news credibility meter. International Journal of Computer Applications, 83(6).

Westerman, D., Spence, P.R. and Van Der Heide, B., 2012. A social network as information: The effect of system generated reports of connectedness on credibility on Twitter. Computers in Human Behavior, 28(1), pp.199-206.

Zhao, Z., Resnick, P. and Mei, Q., 2015, May. Enquiring minds: Early detection of rumors in social media from enquiry posts. In Proceedings of the 24th international conference on world wide web (pp. 1395-1405). 